\title{
Clinical evidence supporting the use of an activated clinoptilolite suspension as an agent to increase urinary excretion of toxic heavy metals
}

This article was published in the following Dove Press journal:

Nutrition and Dietary Supplements

10 November 2009

Number of times this article has been viewed

\author{
James L Flowers' \\ Stewart A Lonky² \\ Erik J Deitsch ${ }^{3}$ \\ 'Eno Research and Development, Inc., \\ Hillsborough, NC, USA; ${ }^{2}$ University of \\ California at Los Angeles, Los Angeles, \\ CA, USA; ${ }^{3}$ Wellness Industries, LLC, \\ Parkland, FL USA
}

\begin{abstract}
Effective treatment of chronic illness resulting from the long-term buildup of heavy metals in the body, such as chelation therapy, presents numerous clinical challenges, including undesirable side effects and unpredictable efficacy. Use of a naturally occurring zeolite, clinoptilolite, to remove these toxic substances may offer an efficacious and safe alternative to the traditional approaches. This study was designed to evaluate the ability of activated clinoptilolite suspended in water (ACS) to remove heavy metals from the body through urinary excretion without the undesirable removal of physiologically important electrolytes. The protocol utilized two treatment groups, each consisting of eleven healthy men aged 36 to 70 years. Volunteers were given a commercially available version of the study substance for seven days (Group 1) and 30 days (Group 2) and urine samples were collected at specified time points in the study. Changes in urinary concentration of the heavy metals were measured by inductively coupled plasma mass spectrometry and compared to the baseline. Also, serum samples were obtained from five individuals in each group and serum electrolytes were measured prior to and after taking the product. Participants in both groups had increased concentrations of heavy metals in the urine with the peak excretion at around day 4. No clinically significant alterations in serum electrolyte levels were seen at either seven or 30 days on ACS. In conclusion, this study demonstrates that the daily use of an activated clinoptilolite suspension represents a potentially safe and effective way to remove toxic heavy metals from the body through increased urinary excretion without removing clinically detrimental amounts of vital electrolytes.
\end{abstract}

Keywords: zeolite, clinoptilolite, heavy metals, toxins, atomic absorption spectroscopy

\section{Introduction}

In the past few years there has been a growing recognition that in contradistinction to the rare occurrence of acute heavy metal toxicity, illness due to the chronic accumulation of metals, such as lead, mercury, arsenic, cadmium, and aluminum is far more common than ever suspected. Because the accumulation of these metals takes place over months or years, there are frequently no acute effects, and because the symptoms are usually quite non-specific, only the astute clinician suspects chronic metal poisoning.

The slow accumulation of heavy metals has been implicated in numerous neurological diseases, including autism and attention-deficit hyperactivity disorder, ${ }^{1,2}$ encephalopathy, ${ }^{3}$ Parkinson's disease, ${ }^{4}$ and Alzheimer's disease. ${ }^{5,6}$ Furthermore, specific links have been made between lead and mercury accumulation and the development of learning disorders other than autism spectrum disorders. ${ }^{7}$ In addition, cardiovascular diseases, such as hypertension and cardiac rhythm disorders, have been
Correspondence: James L Flowers 505 Meadowland Drive, Suite 105, Hillsborough, NC 27278, USA

$\mathrm{Tel}+\mathrm{I} 91924 \mid 4580$

Fax + I $91924 \mid 4584$

Email jamesflowers@enoresearch.com 
linked to the accumulation of lead, ${ }^{8}$ renal disease has been linked to exposures to lead, arsenic, cadmium, and mercury, ${ }^{9}$ and reproductive problems including infertility have been linked to numerous heavy metal exposures. ${ }^{10,11}$

Given these data, it is not surprising that there has been a renewed interest in the various forms of chelation available to help decrease the overall body burden of heavy metals. ${ }^{12,13}$ Unfortunately, some of these chelating agents have significant side effects, including the depletion of needed electrolytes with the potential for cardiac complications and death. ${ }^{14}$ Other chelating agents may be initially effective in removing heavy metals from either the blood or tissue pool, but can then re-deposit these toxic metals in either the brain or the kidney. ${ }^{15}$ Furthermore, long-term use of many of these agents can result in side effects from the chelating agent itself.

The naturally occurring zeolite, clinoptilolite, is a mineral that is known to have unique qualities that might make it an attractive alternative to currently available chelating agents for the removal of heavy metals from blood, tissue, and adipose stores. First of all, like other zeolites it is an aluminosilicate mineral with a unique open lattice structure that results in the mineral having a net negative charge. This negative charge is balanced by an exchangeable cation, usually sodium, potassium or calcium. However, this balancing cation is easily displaced by certain other cations, including metal ions, which have been shown to have a stronger affinity for the clinoptilolite lattice. ${ }^{16,17}$ The internal surface area of clinoptilolite pores that are available for cation exchange is estimated between 10 to $300 \mathrm{~m}^{2} / \mathrm{g}$. ${ }^{18}$ As a result, zeolites in general, and clinoptilolite in particular, have been found to be useful in removing metal contamination from soil ${ }^{19}$ and contaminated water. ${ }^{20}$ In fact, numerous studies have shown that the affinity of clinoptilolite is quite high for some of the most toxic heavy metals. ${ }^{21}$

In concert with these findings in waste and water management, there have been a host of studies demonstrating that clinoptilolite can be fed to animals with beneficial effects. The benefits have been shown to derive from the ability of this zeolite to bind certain toxins in the gut allowing for their removal from the body. ${ }^{22,23}$ This has resulted in improved growth in pigs fed a diet supplemented with zeolites, ${ }^{24,25}$ as well as enhanced reproductive performance of sows. ${ }^{26}$ More recent studies have shown that these beneficial effects occur in the absence of any side effects, even with long-term zeolite supplementation. ${ }^{27}$ These studies have shown that including clinoptilolite in the feed of experimental animals results in no measurable decrease in blood vitamin or mineral content.
There are only scant data available describing the use of zeolites in humans. Maianskaia and colleagues have demonstrated that a zeolite-containing food additive hastened recovery in burn trauma victims, ${ }^{28}$ and Ivkovic and colleagues have shown that a physiochemically enhanced clinoptilolite is capable of acting as an antioxidant and results in the stimulation of immune-competent cells. ${ }^{29}$ In these and other studies, no side effects have been described from the administration of clinoptilolite.

We have reasoned that the heavy metal chelating effects of clinoptilolite that have been shown to occur in vitro might be responsible for some of the beneficial effects seen when this naturally occurring zeolite is administered to animals and humans. An increase in the excretion of heavy metals in subjects who consume clinoptilolite would be an indication that the natural chelating properties of this negatively charged mineral might have clinical applications in cases of metal accumulation and toxicity. We undertook the current study to investigate this hypothesis in normal volunteers who had their diets supplemented with a suspension of activated clinoptilolite for either seven or 30 days, with a measurement of metal excretion at appropriate intervals. Furthermore, we evaluated the effect of this supplementation on the levels of vital serum electrolytes.

\section{Methods}

A total of 33 male subjects aged 35 to 71 years were divided into two experimental groups, group $1(\mathrm{n}=11)$ and group 2 $(n=11)$, and one control group $(n=11)$. Each experimental group consumed a commercially available dietary supplement consisting of an activated clinoptilolite suspension, Natural Cellular Defense ${ }^{\circledR}$ (Waiora, Boynton Beach, FL). The product, a suspension in water, was taken by mouth as 15 drops, twice per day. The control group consumed an equal volume of a placebo solution, prepared as $50 \mathrm{ng} / \mathrm{ml}$ magnesium silicate and $1 \mathrm{mg} / \mathrm{ml}$ citric acid in purified water. Group 1 was evaluated for seven days while group 2 and the control group were evaluated for 30 days. One participant in group 2 submitted a urine sample after 45 days on the activated clinoptilolite suspended in water (ACS) supplement. All participants consulted their personal physicians to insure they had no medical conditions that would preclude participation in this study before reviewing and signing informed consent forms. Inclusion in the study required that participants not be diabetic; not have any serum electrolyte imbalance; not be on any metal-based therapy (such as lithium or gadolinium contrast); not be under therapy for heavy metal toxicity, then or for three months prior; 
and tested positive, above a predetermined threshold value, for at least four of the nine metals in the test panel. Once informed consent was obtained, each participant provided a $50 \mathrm{ml}$ urine sample, taken from the first morning elimination and frozen at $-20^{\circ} \mathrm{C}$. This became the baseline urine sample, referred to as time 0 (T0). Each participant also submitted to a basic metabolic panel for serum electrolytes, creatinine and glucose prior to beginning the study. Five participants from each experimental group and the control group submitted to a second metabolic panel after completing the study.

Each group 1 participant collected $50 \mathrm{ml}$ of urine from the first morning elimination on days 1, 4, and 7. Participants in group 2 collected $50 \mathrm{ml}$ of urine from the first morning elimination on days $1,4,7,10,14,21$, and 30 . The control group collected $50 \mathrm{ml}$ of urine from the first morning elimination on days 1, 10, 20, and 30. For all samples, the urine was immediately placed in pre-labeled polypropylene tubes and frozen at $-20{ }^{\circ} \mathrm{C}$ until the end of the study.

At the end of the study, each urine sample was thawed on ice and then divided into three aliquots. First, for all metals except mercury, urine was diluted 1:1 with a solution of $15 \%$ $\mathrm{HNO}_{3}$ in ultrapure $\mathrm{H}_{2} \mathrm{O}$ and containing yttrium as an internal standard (S1). For mercury analysis, a second sample was prepared by diluting the urine $1: 1$ with a solution of $15 \%$ $\mathrm{HCl}, 0.25 \%$ cysteine and $1.75 \%$ EDTA with yttrium as an internal standard (S2). The final sample remained unaltered (S3) and was utilized for urinary creatinine analysis. A fourth sample, the ACS product itself, was also subjected to acid extraction and subjected to trace metal analysis using the same protocols.

Heavy metal content was measured by inductively coupled plasma mass spectroscopy (ICP-MS) using a SCIEX 6100 DRC (PerkinElmer, Wellesley, MA) using standard methods. Calibrations of the instrument for each element were performed with four-point calibration curves and forced through zero. Briefly, $5 \mathrm{ml}$ of the product was aspirated into the ICP-MS analyzer and concentrations of analytes ascertained using calibration curves. Two control samples carried through the same processes as the test samples were used to validate the calibration. Each measurement was repeated three times.

Measurements of urinary creatinine levels and serum electrolyte levels were performed in a commercial clinical laboratory using standard protocols and reagents. Urinary creatinine results were expressed as $\mathrm{mg} / \mathrm{dL}$, serum creatinine levels were expressed as $\mathrm{mmol} / \mathrm{L}$, serum glucose concentration was expressed as $\mathrm{mg} / \mathrm{dL}$ and serum electrolyte concentrations were expressed as $\mathrm{mmol} / \mathrm{L}$.
Clinoptilolite, as it is mined, contains dirt and numerous chemical contaminants, including many of the heavy metals being evaluated in this study. The process laboratory (Mineral Sciences, LLC, Hillsborough, NC) that produces and provides the activated clinoptilolite used in the manufacture of Natural Cellular Defense ${ }^{\circledR}$ treats the raw mineral using a proprietary, multi-step process consisting of mechanical reduction of particle size to a range of $0.5 \mu \mathrm{m}$ to $5 \mu \mathrm{m}$ to increase the surface area, followed by a series of complex, temperature-controlled ion exchanges, coupled with numerous washes and filtrations, to remove these naturally occurring impurities. Samples of both the raw and the processed clinoptilolite mineral were obtained and analyzed by powder diffraction and electron microscopy, using standard methods and algorithms, ${ }^{30}$ to verify that the mineral in Natural Cellular Defense ${ }^{\circledR}$ was in fact clinoptilolite and that the activation process did not alter the composition of the zeolite mineral.

\section{Results and discussion}

Analysis of participant age, serum electrolyte levels, serum creatinine levels and urinary creatinine levels using ANOVA revealed that there were no significant differences between or among the experimental groups and the control group on any of the baseline parameters prior to beginning the study (Table 1).

Individuals also submitted to a toxic metals urinalysis prior to being selected to participate in the study. Participants selected for inclusion in the study tested positive for at least four, and as many as seven, metals in the baseline, pre-screen urinalysis (aluminuim $[\mathrm{Al}]=6$, antimony $[\mathrm{Sb}]=3$, arsenic $[\mathrm{As}]=7$, bismuth $[\mathrm{Bi}]=3$, cadmium $[\mathrm{Cd}]=8$, lead $[\mathrm{Pb}]=8$, mercury $[\mathrm{Hg}]=8$, nickel $[\mathrm{Ni}]=3$, tin $[\mathrm{Sn}]=3$ ). In order to detect and evaluate both positive and negative fluctuations in urinary metal excretion, inclusion criteria also required each selected participant to excrete at least the minimum defined levels of toxic metals into the urine prior to use of the ACS supplement. The levels were established as Al, 70; $\mathrm{Sb}$, 1.5; As, 50; Bi, 0.5; Cd, 2.25; Pb, 170; Hg, 7.5; Ni, 30; and $\mathrm{Sn}, 7 \mu \mathrm{g} / \mathrm{g}$. Metal concentrations were normalized to creatinine, which is excreted at a steady rate into the urine, to compensate for the concentration of water in the urine and reduce dilutional effects.

Prior to initiation of the study, ICP-MS trace metals analysis was conducted on the commercial ACS supplement to determine if the product itself was contaminated with sufficient concentrations of the 10 heavy metals being screened for in this study to affect the outcome of the urinary excretion analysis. The analysis revealed insignificant concentrations of heavy 
Table I Comparison of baseline parameters between and among experimental and control groups

\begin{tabular}{|c|c|c|c|}
\hline & Group I & Group 2 & Control \\
\hline Parameter & $\mathrm{n}=\mathrm{II}$ & $\mathrm{n}=\mathrm{II}$ & $\mathrm{n}=\mathrm{II}$ \\
\hline Age, years (SD) & $52.7(10.14)$ & $53.3(10.15)$ & $53.9(10.65)$ \\
\hline Urine creatinine, mg/dL (SD) & $429.5(117.6)$ & $389.9(149.3)$ & $421.2(158.2)$ \\
\hline \multicolumn{4}{|l|}{ Serum electrolyte panel } \\
\hline Creatinine, mmol/L (SD) & $82.1(11.70)$ & $84.8(10.43)$ & $87.6(9.02)$ \\
\hline Glucose, mg/dL (SD) & $78.3(|0.3|)$ & $76.3(9.20)$ & $74.4(7.42)$ \\
\hline Potassium, mmol/L (SD) & $4.3(0.48)$ & $4.4(0.39)$ & $4.3(0.29)$ \\
\hline Magnesium, mmol/L (SD) & $2.24(0.29)$ & $2.31(0.28)$ & $2.28(0.30)$ \\
\hline Calcium, mmol/L (SD) & $9.13(0.31)$ & $8.93(0.40)$ & $9.01(0.39)$ \\
\hline Sodium, mmol/L (SD) & I 42.7 (4.49) & $14 \mid .1(3.91)$ & $140.5(3.22)$ \\
\hline Chloride, mmol/L (SD) & $102.5(3.08)$ & I0I.4 (2.69) & $101.2(1.93)$ \\
\hline
\end{tabular}

Notes: Statistical analysis of the baseline laboratory values for the two experimental groups and the control group show no significant difference in starting parameters. Values are in units as listed in the tables with standard deviations (SD).

metals in the ACS supplement, most below limits of detection. Data from the powder diffraction/electron microscopy analysis unambiguously identified the mineral in the supplement as clinoptilolite, primarily in the form of calcium aluminosilicate, with potassium, magnesium, and sodium cations present.

Since the purpose of this study was not to quantify urinary excretion of heavy metals but to evaluate changes in overall excretion following dietary supplementation with ACS, the urinary concentration of heavy metals measured during the study were converted to a relative concentration compared to baseline urinary concentration (time point value/baseline value) for each metal measured. The mean value for all metals was then calculated for each urine collection time point and plotted against time. Using analysis of variance, the variance between the time points was evaluated.

Individuals in the control group did not exhibit statistically significant changes in urinary excretion of heavy metals during the evaluation period, although insignificant fluctuations, both positive and negative, were detected. To establish the dynamics of an ACS-induced increase in heavy metal excretion, the changes in measured excretion for each heavy metal analyte for all participants were averaged and plotted against time. As shown in Figure 1, group 1 participants exhibited significantly increased urinary excretion of the studied analytes measured on days 1,4 , and 7 compared to day $0\left(P=1.2 \times 10^{-8}\right)$ and compared to all measurements of the control group. During the study, day 4 excretion levels were generally greater than that observed on days 1 and 7; however, no statistical difference exists between days 1 and $7(P=0.909)$.

The excretion profile of a single participant in group 1 is presented in Figure 2. This individual began the study with the highest baseline concentrations of $\mathrm{Sb}, \mathrm{Hg}$, and $\mathrm{Pb}$. Although the dynamics of ACS-induced heavy metal excretion was similar to the mean of the group presented in Figure 1, a delay in the reduction of excretion towards baseline for $\mathrm{Hg}$ and $\mathrm{Pb}$ was observed for this individual. This observation was primarily attributed to the higher baseline burden of $\mathrm{Hg}$ and $\mathrm{Pb}$ carried by the participant. Additionally, the mechanisms of excretion and sequestration for these metals are also dissimilar and could contribute to the variance. Still, this result typifies the heterogeneous nature of the excretion phenomenon. Across the whole of Group 1 and for each individual analyte, both time to peak excretion and magnitude of excursion from baseline excretion were unique to each participant.

In the 30-day test group, mean toxic metal excretion was increased on days $1,4,7,14$ and 21 when compared to that observed on day $0(P=0.037)$, with most peaking at the four-day measurement (Figure 3). Participants taking the placebo substance had no increase in urinary heavy metal excretion during the course of the study, however all participants in Group 2 exhibited statistically significant enhancement of urinary toxic metal clearance on days $1(P=0.021)$, $4(P=0.007), 7(P=0.010)$, and $14(P=0.030)$ time points when compared to placebo control. When compared to both baseline measurements for group 2 and same time point value for the control group, five members of group 2 continued to excrete increased levels of heavy metals at 21 days; however, the mean of the group for this point was not statistically different from either the baseline value for group $2(P=0.81)$ or the control group value $(P=0.23)$.

The urinalyses of 10 of 11 group 2 subjects demonstrated mean excretion concentrations of toxic metals on day 30 to 


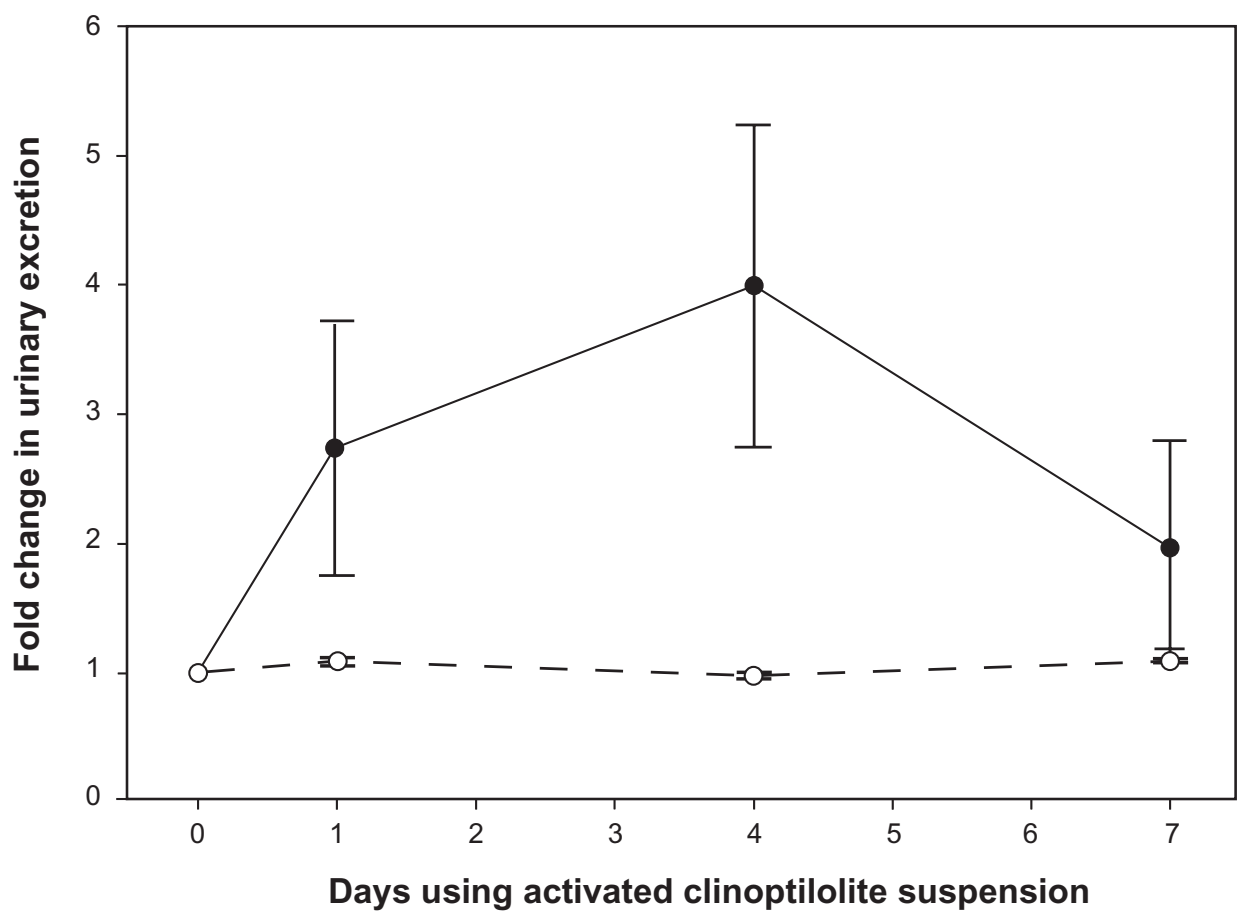

Figure I Fold change in urinary excretion of all heavy metal analytes for group I over seven days as compared to placebo control group. Notes: Data points represent a compiled average of the fold changes measured for urinary excretion of all heavy metal analytes evaluated in each participant in group I (•) and the placebo control group (O) at days $0,1,4$, and 7. Error bars represent the standard deviation of the compiled averages. "Fold change in urinary excretion" is defined as the level of urinary excretion of metal toxins measured by ICP-MS prior to initiating ACS supplementation divided by the level of urinary excretion of metal toxins measured at specified time point intervals.

Abbreviations: ACS, activated clinoptilolite suspended in water; ICP-MS, inductively coupled plasma mass spectrometry.

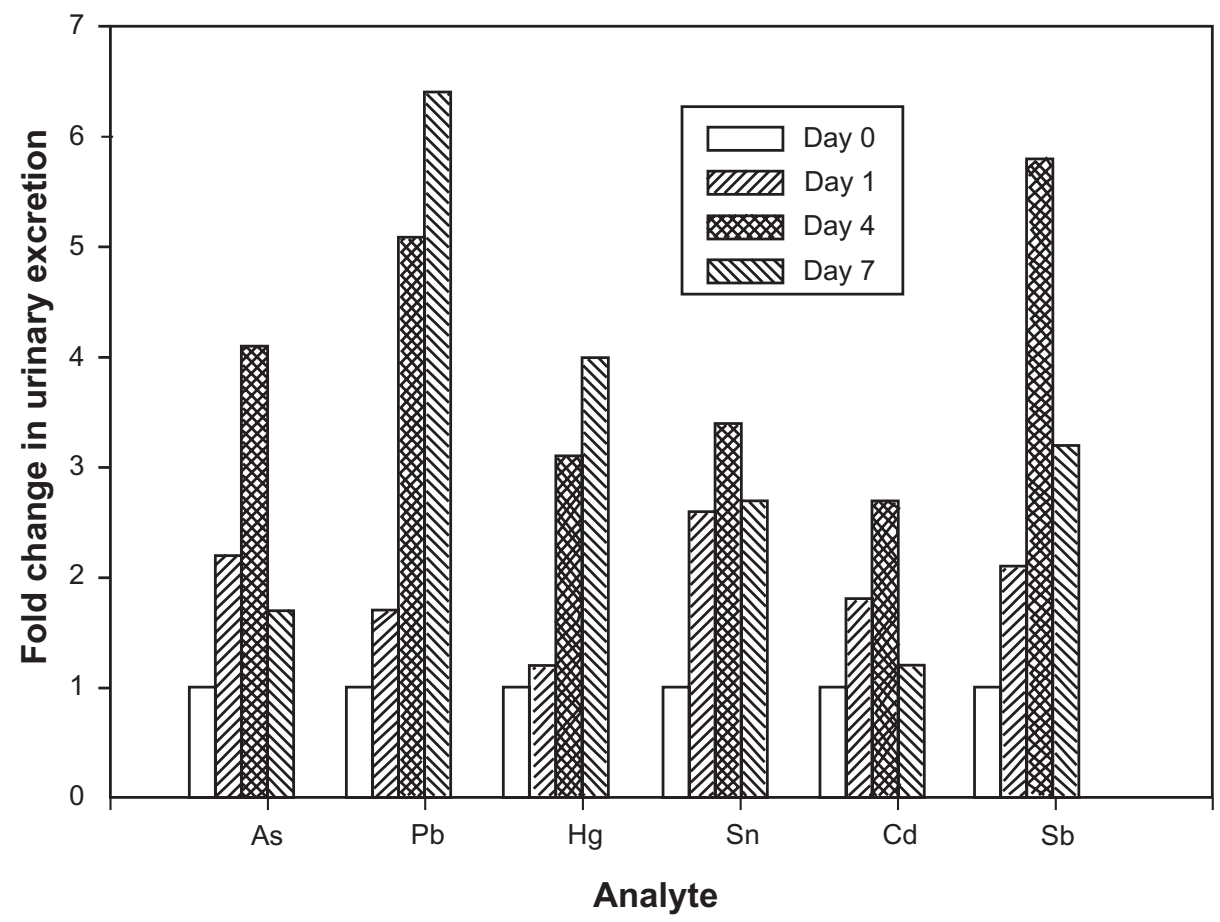

Figure 2 Heavy metal urinary excretion profile for an individual group I participant.

Notes: Bars represent the fold change in excretion of the labeled analytes on days $0,1,4$, and 7 . "Fold change in urinary excretion" is defined as the level of urinary excretion of metal toxins measured by ICP-MS prior to initiating ACS supplementation divided by the level of urinary excretion of metal toxins measured at specified time point intervals. Abbreviations: ACS, activated clinoptilolite suspended in water; ICP-MS, inductively coupled plasma mass spectrometry. 


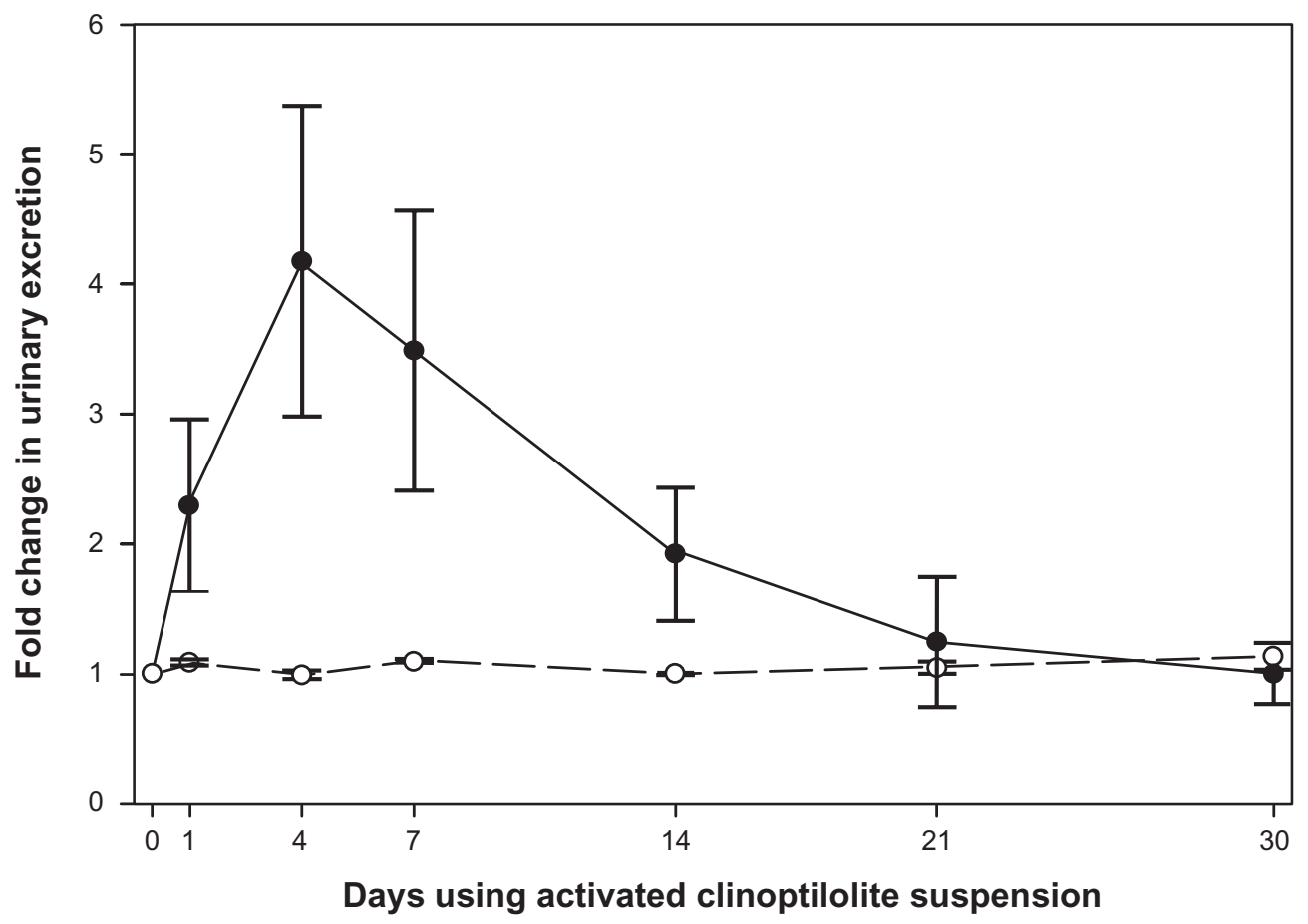

Figure 3 Fold change in urinary excretion of all heavy metal analytes for group 2 over 30 days as compared to the placebo control group.

Notes: Data points represent a compiled average of the fold changes measured for urinary excretion of all heavy metal analytes evaluated in each participant in group 2 ( $\bullet$ ) and the placebo control group (O) at days $0,1,4,7,14,21$, and 30. Error bars represent the standard deviation of the compiled averages. "Fold change in urinary excretion" is defined as the level of urinary excretion of metal toxins measured by ICP-MS prior to initiating ACS supplementation divided by the level of urinary excretion of metal toxins measured at specified time point intervals.

Abbreviations: ACS, activated clinoptilolite suspended in water; ICP-MS, inductively coupled plasma mass spectrometry.

be at or below day 0 levels $(P=0.02$; Figure 3$)$, with one participant exhibiting 30 -day levels significantly higher than day $0(P=0.002)$. Follow-up analysis of this individual after 45 days of using the ACS product revealed urinary heavy metal levels equivalent to or below the initial excretion levels observed prior to initiating ACS. This is consistent with the idea of heterogeneous excretion profiles and responses to taking ACS, based on individual physiology and circumstances.

Analysis of the serum electrolyte panels collected prior to and after the study revealed no significant changes from creatinine, glucose, potassium, magnesium, calcium, sodium, or chloride baseline levels (Table 2) following use of the activated clinoptilolite suspension for seven days. The 30-day and the control groups also remained unchanged.

\section{Conclusion}

In this study, we have presented clinical evidence supporting the use of an activated clinoptilolite suspension to safely and effectively increase the urinary excretion of potentially toxic heavy metals in healthy volunteers without negatively impacting the electrolyte profiles of the participants.
Significant increases in the urinary excretion of aluminum, antimony, arsenic, bismuth, cadmium, lead, mercury, nickel and tin were observed in the subjects participating in the two study groups as compared to placebo controls. Since spectroscopic analysis showed no detectable contamination of the activated clinoptilolite suspension with the metals being evaluated, it is reasonable to assume that this observation was attributable to increased excretion of stored toxins and certainly led to a reduction in the overall toxic metal body burden of the participants.

It is notable that the degree of response, as well as relative timing of the response, to the ACS supplement was variable among participants. This was not completely unexpected and can be explained by a number of variables, from the innate differences in physiology among the individuals within each group to differing levels of compliance with the instructions for taking the ACS supplement. However, for all participants of this study, consumption of an ACS supplement for either seven or 30 days resulted in a measurable enhancement of urinary excretion of heavy metals in subjects that demonstrated a positive excretion phenotype by testing positive for those toxins in the prescreening urinalysis. The kinetics of 
Table 2 Serum electrolyte levels following seven- and 30-day use of ACS

\begin{tabular}{|c|c|c|c|}
\hline \multirow[t]{2}{*}{ Electrolyte panel } & Group I & Group 2 & $\begin{array}{l}\text { Control } \\
\text { group }\end{array}$ \\
\hline & $\mathbf{n}=\mathbf{5}$ & $\mathbf{n}=\mathbf{5}$ & $\mathbf{n}=\mathbf{5}$ \\
\hline Creatinine, mmol/L (SD) & $84.6(5.33)$ & $81.9(9.63)$ & $83.6(9.59)$ \\
\hline Glucose, mg/dL (SD) & $75.5(4.32)$ & $73.2(5.72)$ & $83.1(|5.7|)$ \\
\hline Potassium, mmol/L (SD) & $4.2(C$ & 4.4 & $4.2(0.33)$ \\
\hline Magnesium, mmol/L (SD) & $2.26(0.26)$ & $2.40(0.21)$ & $2.32(0.24)$ \\
\hline Calcium, mmol/L (SD) & $8.99(0.21)$ & $9.4 \mathrm{I}(0.20)$ & $9.34(0.27)$ \\
\hline Sodium, mmol/L (SD) & $140.9(3.26)$ & $142.0(2.67)$ & $141.0(1.97)$ \\
\hline Chloride, mmol/L (SD) & $102.1(2.76)$ & $102.1(1.09)$ & $101.8(2.11)$ \\
\hline
\end{tabular}

Notes: Statistical analysis of serum electrolyte values for five members from each of the two experimental groups and the control group exhibit normal range levels following dietary supplementation with ACS. Values are in units as listed in the tables with standard deviations (SD) in parentheses.

Abbreviation: ACS, activated clinoptilolite suspended in water.

excretion observed in this study, which revealed a several-fold increased peak in urinary excretion of toxic metals at four days for both groups taking ACS, implies that the supplement may serve initially to help clear free, exchangeable heavy metal cations from the body, while the continued excretion of these toxic species, albeit at reduced levels, out to 14 days, and in some cases out to 21 and even 30 days, suggests that use of an ACS supplement for a longer time may result in removal of sequestered toxins from various tissues.

Across the globe, heavy metal toxins and other pollutants are ubiquitous in our environment. These pollutants exist in our air, water, and food supplies, our workplaces, even our homes and automobiles. The human physiological system has many mechanisms in place for excreting these contaminants, through sweating, incorporation into hair and nails, defecation and urination. However, the ability to excrete and eliminate these environmental toxins is highly variable by individual phenotype as well as the nature of the contaminant. As such, these toxins are also metabolized and processed to render them "less toxic" through processes including sequestration in adipose, brain and muscle tissue. This buildup often occurs very slowly and can eventually result in clinically significant levels of heavy metals that result in disease.

The increased awareness of the role of chronic heavy metal poisoning in the clinical manifestation of a broad array of disorders and syndromes has resulted in an increased focus on therapies that remove or reduce these toxins. The use of activated clinoptilolite suspension to remove these ubiquitous toxins from the body presents an alternate modality of therapy to traditional treatment through chelation. Additionally, the use of a safe and natural product like activated clinoptilolite suspension may provide a solution to the many challenges presented by the more traditional therapies, such as the requirement for extended time spent in a medical facility and the associated costs incurred; undesirable side effects; and unpredictable efficacy. Further studies using activated clinoptilolite suspension in individuals suffering both chronic and acute exposure to high levels of heavy metal toxins, from environmental, occupational and lifestyle-associated sources, is warranted.

\section{Acknowledgments}

The authors report the following potential conflicts of interest. James L Flowers is a consultant to the company that manufactures activated clinoptilolite. Erik J Deitcsh is employed by the company that manufactures Natural Cellular Defense ${ }^{\circledR}$. Stewart A Lonky has no conflicting interests. This work was supported by a corporate grant from Wellness Industries, LLC.

\section{References}

1. Mutter J, Naumann J, Schneider R, et al. Mercury and autism: accelerating evidence? Neurendorinol Lett. 2005;26:439-446.

2. Windham G, Zhang L, Gunier R, et al. Autism spectrum disorders in relation to distribution of hazardous air pollutants in the San Francisco Bay area. Environ Health Perspect. 2006;114:1438-1444.

3. Berbel-Garcia A, Gonzale-Aguirre JM, Botia-Paniagua E, et al. Acute polyneuropathy and encephalopathy caused by arsenic poisoning. Rev Neurol. 2004;38:928-930.

4. Coon S, Stark A, Peterson E, et al. Whole-body lifetime occupational lead exposure and the risk of Parkinson's disease. Environ Health Perspect. 2006;114:1872-1876.

5. Walton JR. Aluminum in hippocampal neurons from humans with Alzheimer's disease. Neurotoxicology. 2006;27:385-394.

6. Grant WB, Campbell A, Itzhaki RF, Savory J. The significance of environmental factors in the etiology of Alzheimer's disease. J Alzheimers Dis. 2002;4:179-189.

7. Lewis M, Worobey J, Ramsay DS, et al. Prenatal exposure to heavy metals: effect on childhood cognitive skills and health status. Pediatrics. 1992;86:1010-1015.

8. Navas-Acien A, Gualler E, Silbergeld EK, Rothenberg SJ. Lead exposure and cardiovascular disease - a systematic review. Environ Health Perspect. 2007;115:472-482.

9. de Burbure C, Buchet JP, Leroyer A, et al. Renal and neurological effects of cadmium, lead, mercury, and arsenic in children: evidence for early effect and multiple interactions at the environmental exposure levels. Environ Health Perspect. 2004;114:584-590.

10. Shiau CY, Wang JD, Chen PC. Decreased fecundity among male lead workers. Occup Environ Med. 2004;61:915-923.

11. Olfert SM. Reproductive outcomes among dental personnel: a review of selected exposures. J Can Dent Assoc. 2006;72:821-825.

12. Kosnett MJ, Wedeen RP, Rothenberg SJ, et al. Recommendations for medical management of adult lead exposure. Environ Health Perspect. 2007;115:463-471.

13. Andersen $\mathrm{O}$. Chemical and biological considerations in the treatment of metal intoxications by chelating agents. Mini Rev Med Chem. 2004;4:11-21.

14. Centers for Disease Control and Prevention (CDC). Deaths associated with hypocalcemia from chelation therapy - Texas, Pennsylvania, and Oregon, 2003-2005. MMWR Morb Mortal Wkly Rep. 2006;55:204-207. 
15. Flora SJ, Flora G, Saxena G, et al. Arsenic and lead induced free radical generation and their reversibility following chelation. Cell Mol Biol. 2007;53:26-47.

16. Bailey S, Olin TJ, Bricka RM, et al. A review of potentially lo-cost sorbant for heavy metals. Water Res. 1999;33:2469-2479.

17. Sideheswaran $P$, Bhat AN. Impact of zeolite water content on exchange of calcium ions. Thermochimica Acta. 1997;298:55-58.

18. Korkuna O, Leboda R, Skubiszewska-Zieba J, et al. Structural and physiochemical properties of natural zeolites: clinoptilolite and mordenite. Microporous Mesoporous Mater. 2006;87:243-254.

19. Oste LA, Lexmond TM, Van Reimsdijk WH. Metal immobilization in soils using synthetic zeolites. J Environ Qual. 2002;31:813-821.

20. Pitcher SK, Slade RC, Ward NI. Heavy metal removal from motorway stormwater using zeolites. Sci Total Environ. 2004;134:161-166.

21. Sprynskyy M, Buszewski B, Terzyk AP, et al. Study of the selection mechanism of heavy metal $\left(\mathrm{Pb} 2^{+}, \mathrm{Cu} 2^{+}, \mathrm{Ni} 2^{+}\right.$and $\left.\mathrm{Cd} 2^{+}\right)$adsorption on clinoptilolite. J Colloid Interface Soc. 2006;304:21-28.

22. Spotti M, Fracchiolla ML, Arioli F, et al. Aflatoxin B1 binding to sorbants in bovine ruminal fluid. Vet Res Commun. 2005;29:507-515.

23. Kransnoperova AP, Lonin A. Effects of natural zeoite-clinoptilolite on process of removal of Cs-137 from the rat body. Radiats Biol Radioecol. $1999 ; 39: 471-474$.
24. Pond Wg, Yen JT, Varel VH. Response of growing swine to dietary copper and clinoptilolite. Bull Environ Contamin Toxicol. 1988;37: 795-803.

25. Coffey MT, Picklington DW. Effect of feeding zeolite-A on the performance and carcass quality of swine. J Animal Sci. 1989;67:36.

26. Mumpton FA, Fishman PH. The application of natural zeolites in animal science and aquaculture. J Animal Sci. 1977;45:1188-1203.

27. Papaioannou SC, Kyriakis SC, Papasteriadis A, et al. Effect of in-feed inclusion of a natural zeolite (clinoptilolite) on certain vitamin, macro and trace element concentration in the blood, liver and kidney tissues of sows. Res Vet Sci. 2002;72:61-68.

28. Maiankaia NN, Blagitko EM, Poliakovitch AS, et al. Use of zeolitecontaining biologically active food supplement in patients with burn trauma. Vopr Pitan. 2004;73:24-27.

29. Ivkovic S, Deutsch U, Silberbach A, et al. Dietary supplementation with tribomechanically activated zeolite clinoptilolite in immunodeficiency: effects on the immune system. Adv Ther. 2004;21:135-147.

30. Gramm F, Baerlocher C, McCusker LB, et al. Complex zeolite structure solved by combining powder diffraction and electron microscopy. Nature. 2006;444:79-81.
Nutrition and Dietary Supplements

\section{Publish your work in this journal}

Nutrition and Dietary Supplements is an international, peer-reviewed, open access journal focusing on research into nutritional requirements in health and disease, impact on metabolism and the identification and optimal use of dietary strategies and supplements necessary for normal growth and development. The journal welcomes papers covering

\section{Dovepress}

original research, basic science, clinical \& epidemiological studies, reviews and evaluations, guidelines, expert opinion and commentary, case reports and extended reports. The manuscript management system is completely online and includes a very quick and fair peer-review system, which is all easy to use.

Submit your manuscript here: http://www.dovepress.com/nutrition-and-dietary-supplements-journal 